Pacific Journal of Mathematics

POTENTIAL OPERATORS AND EQUIMEASURABILITY 


\title{
POTENTIAL OPERATORS AND EQUIMEASURABILITY
}

\author{
A. N. Al-HussainI
}

\section{W. Rudin proved the following.}

Theorem 1.1. Assume $0<p<\infty, p \neq 2,4,6, \cdots$. Let $n$ be a positive integer. If $f_{i} \in L_{p}(\mu), g_{i} \in L_{p}(\nu)$ for $1 \leqq i \leqq n$ and

$$
\int_{X}\left|1+z_{1} f_{1}+\cdots+z_{n} f_{n}\right|^{p} d \mu=\int_{Y}\left|1+z_{1} g_{1}+\cdots+z_{n} g_{n}\right|^{p} d \nu
$$

for all $\left(z_{1}, \cdots, z_{n}\right) \in C^{n}$, then $\left(f_{1}, \cdots, f_{n}\right)$ and $\left(g_{1}, \cdots, g_{n}\right)$ are equimeasurable. Here as usual $L_{p}(\mu)$ and $L_{p}(\nu)$ stand for $p$ th power integrable functions defined on finite measure spaces $(X, X, \mu)$ and $(Y, Y, \nu)$ respectively. $\mathscr{C}$ is the field of complex numbers.

The purpose of this paper is to provide a new setting for Rudin's result by recasting it and its extension to real valued functions into the framework of the theory of potential operators as formulated by $\mathrm{K}$. Yosida.

We begin by outlining the theory of potential operators ([8], [9]). K-I. Sato's 1970 paper [6] contains extensive material on the subject.

Let $T_{t}$ be a strongly continuous semigroup of linear operators on a Banach space $X$, satisfying $\sup _{t}\left\|T_{t}\right\|<+\infty$, with infinitesimal generator $A=s \lim _{t \rightarrow 0}\left(T_{t} f-f\right) / t$ where as usual $s$ denotes the strong limit, and resolvent $J_{\lambda}=(\lambda-A)^{-1}, \lambda>0$. K. Yosida defined the potential operator $V$ as follows:

$$
V f=s \lim _{\lambda \rightarrow 0} J_{\lambda} f,
$$

if the strong limit exists for a dense set in $X$. This is one way to unify the potential operator concept for a large class of Markov processes, which includes Brownian motion, stable processes and of course transient Markov processes.

Motivated by an application to equimeasurability in Section (3), we specialize to potential operators induced by Markov processes. Thus let $S$ be a locally compact, noncompact, Hausdorff space with countable basis. By $C_{0}(S), C_{k}(S)$ we denote the spaces of real valued functions which vanish at infinity, and those with compact support respectively. Let $T_{t}$ be a strongly continuous semigroup of positive linear operators on $C_{0}(S)$ with $\left\|T_{t}\right\| \leqq 1$. To this semigroup there corresponds a right continuous Markov process $\left\{X_{t}\right\}$ on $S$ with transition $P_{t}(x, A)$, such that:

$$
T_{t} f(x)=\int P_{t}(x, d y) f(y) .
$$


We will not distinguish between $T_{t}$ and its corresponding Markov process.

If $\int_{0}^{+\infty} P_{t}(x, K) d t<+\infty$ for all $x \in S$, all compact sets $K$, we call the Markov process transient. If $\int_{0}^{+\infty} P_{t}(x, \mathcal{O}) d t=+\infty$ for all $x \in S$, and all open sets $\mathcal{O}$, we call the Markov process recurrent. It will be called null recurrent if it is recurrent and $\lim _{t \rightarrow \infty} P_{t}(x, K)=0$ for all $x \in S$, all compact sets $K . \quad V$ the potential operator introduced earlier in this section exists for transient and null recurrent Markov processes. (Theorem 3.1, Theorem 3.2 [6].) We will make use of the following theorem, whose proof is included in Theorem 2.3 of [6].

THEOREM 1.2. If a semigroup $T_{t}$ admits a potential operator $V$, then $\mathscr{D}(V)=\mathscr{R}(A), \mathscr{R}(V)=\mathscr{D}(A)$, and $V=-A^{-1}$. Here $\mathscr{D}$ and $\mathscr{R}$ stand for domain and range respectively.

2. More about Markov processes. Concepts treated in this section are mainly those necessitated by the section to follow. Let $\left\{X_{t}\right\}$ be a Markov process on $S$ (as in $\S 1$ ), with transition $P_{t}(x, A)$ which as assumed to exist. As is well known,

$$
J_{\lambda} f=\int_{0}^{+\infty} e^{-\lambda t} T_{t} f d t, \quad \text { where } \quad T_{t} f=\int P_{t}(x, d y) f(y) .
$$

Set $P_{t, \lambda}(x, A)=e^{-\lambda t} P_{t}(x, A)$. Then there is a Markov process $\left\{X_{t, \lambda}\right\}$, a sub-Markov process of $\left\{X_{t}\right\}$, corresponding $P_{t, \lambda}(x, A)$. Thus $V$ may be viewed as a limit of potentials $\int_{0}^{+\infty} T_{t, \lambda} f d t$ where $T_{t, \lambda}$ is the semigroup associated with $\left\{X_{t, \lambda}\right\}$. Also we may note that the infinitesimal generator $A_{\lambda}$ of $T_{t, \lambda}$ is given by $A_{\lambda}=A-\lambda$, (see [2]). By Theorem 1.2, there is a $\phi \in \mathscr{D}(V)$ for every $f \in \mathscr{D}(A)$, such that $f=V \phi$ or alternatively, by making use of $\left\{X_{t, \lambda}\right\}, \lambda>0$, a subMarkov process of $\left\{X_{t}\right\} ; A_{\lambda} f=-\left(-A_{\lambda} f\right)$ we obtain $f=V_{\lambda}\left(-A_{\lambda} f\right)$, using ([2], pp. 24) where $V_{\lambda}$ is the potential operator of $\left\{X_{t, \lambda}\right\}$. Taking limit as $\lambda \rightarrow 0, f=V(-A f)=V \phi$.

Some known examples of $V$ are [1], [3], [4], [6].

ExAmple (1). One-dimensional Brownian motion:

$$
V f(x)=c \int|x-y| f(y) d y .
$$

Example (2). One-dimensional stable process of order $0<\alpha<1$, $1<\alpha<2$. Here $V f=c \int|x-y|^{\alpha-1} f(y) d y$.

ExAmple ( 3 ). n-dimensional stable process. 


$$
V f(x)=c \int|x-y|^{\alpha-n} f(y) d y, \quad 0<\alpha<2,0<\alpha<n .
$$

The constants $c$ are not the same from line to line. Example (1) could be included in Example (2) if we allow $\alpha=2$. Example (3), with additional probabilistic arguments will be used ( $\$ 3$ ) to give an alternative proof to Theorem (1) of [5] concerning equimeasurability of $C^{n}$ valued random functions. W. Rudin proves his theorem by transforms and techniques based on complex variable theory.

By utilizing Examples (1) and (2), we will give an $\mathscr{R}^{n}$ version of the theorem cited above.

The reader may observe that $V f$ 's in the preceding examples are none other than Riesz potentials. Within general theory of Markov processes $V f$ is just $\iint P_{t}(x, y) f(y) d t d y$ the Green's potential, if the Markov process is transient $\left(p_{t}(x, y)\right.$ is the transition density). However if the Markov process is null recurrent and transition density $p_{t}(x, y)$ exists then $V f$ is the limit of potentials of some sub-Markov processes. More precisely $V f=\lim _{\lambda \rightarrow 0} V_{\lambda} f$, where $V_{\lambda}$, $\lambda>0$ is the Green potential corresponding to $e^{-\lambda t} p_{t}(x, y)$.

One-dimensional stable process $0<\alpha<2$, require special attention. $V$ (the set of all functions in $C_{k}(S)$ having null integrals) is dense in $C_{0}(S)$ ([4], [6]). Another important matter is the core of $V$. A set $M$ is called core for $V$ [6] if $M \subset \mathscr{D}(V)$ and if the smallest closed extension of $V \mid M$ coincides with $V$. If $M$ is a core for $V$, then $M$ is dense in $\mathscr{D}(V)$, and $V \mid M$ determines the semigroup. Also $V(M)$ is dense in $\mathscr{R}(V)$. In [6], see also [4], it is shown that $C_{k}(S) \cap \mathscr{D}(V)=M_{0}$ is a core for $V$.

To extend this to $C_{k}^{+\infty}\left(R^{N}\right)$, the set of all infinitely differentiable functions on $R^{N}$, (Theorem 2.3), we borrow (for convenience) some results from [7].

We say a function $\phi$ is $\alpha$ order homogeneous on $R^{N}$, outside a compact set, if there is a $b>0$ such that

$$
\phi(\lambda x)=\lambda^{\alpha} \phi(x) \text { for }|x| \geqq b, \quad \lambda \geqq 1 .
$$

Define $\tilde{\phi}(x)=(|x| / b)^{\alpha} \phi(b x /|x|)$ and note $\phi(x)=\tilde{\phi}(x)$ for $|x| \geqq b$.

THEOREM 2.1. If $X_{t}$ is a transient process with stationary independent increment, with right continuous paths and $E\left|X_{t}\right|^{\alpha}<+\infty$ for a real number $\alpha>0$. Let $\phi_{i}, 1 \leqq i \leqq l$, be an arbitrary number of continuous functions on $R^{N}$ such that $\phi_{i}$ is $\alpha_{i}$ order homogeneous outside a compact set, $0<\alpha_{i} \leqq \alpha$ and such that the set $\left\{\phi_{i}: 1 \leqq i \leqq l\right\}$ is linearly independent. Given real numbers $a_{i}, 1 \leqq i \leqq l$, let $\mathscr{C}$ be the set of function $f \in C_{k}^{+\infty}\left(R^{N}\right)$ such that 


$$
\int_{R^{N}} f(x) d x=0, \int_{R^{N}} f\left((x) \phi_{i}(x) d x=a_{i}, \quad 1 \leqq i \leqq l .\right.
$$

Then $\mathscr{C}$ is a core of the potential $V$.

Proof. See Theorem 5.1 [7].

For the next theorem, we assume for some $t$ the distribution of $X_{t}$ has a nontrivial absolutely continuous part, and that $X_{t}$ is recurrent stationary independent process with right continuous paths, in which case $N=1$ or 2 .

THEOREM 2.2. If $E\left|X_{t}\right|<\infty$, then the set $f \in C_{k}^{\infty}\left(R^{N}\right)$ satisfying

$$
\int_{R^{N}} f(x) d x=\int_{R^{N}} f(x) x_{i} d x=0 \text { for } 1 \leqq i \leqq N
$$

is a core of the potential operator $V$.

Proof. See Theorem 6.1 [7].

For the next theorem $N=1$ or 2 only.

THEOREM 2.3. For a stable process $X_{t}$ with index $0<\alpha<2$, $(\alpha \neq 1, N=1) ; C_{k}^{\infty} \cap \mathscr{D}(V)$ is a core of the potential operator $V$.

Here and elsewhere, it is understood that we are dealing with right continuous path versions.

Proof. We divide the proof into 2 cases:

Transient Case. $(0<\alpha<1, N=1)$ or $(0<\alpha<2, N=2)$. Let $\varepsilon>0$ be such that $\alpha-\varepsilon>0$ and take $\phi(x)=\lambda^{\alpha-\varepsilon-1} x, x \in R^{N}$. The proof is completed by Theorem 2.1. It may be remarked that the introduction of the $\varepsilon$ above was to insure $E|X|^{\alpha-\varepsilon}<+\infty$, by moment properties of stable processes.

Recurrent Case. $(1<\alpha<2, N=1)$. Here again $E\left|X_{t}\right|<+\infty$ by moment properties of stable processes, since $1<\alpha$. The rest follows from Theorem 2.2.

We did not consider $\alpha=1, N=1$. Instead we follow Example (1), a detour.

3. Equimeasurability. Let $\left(\Omega_{1}, \mathscr{A}_{1}, P_{1}\right)$ and $\left(\Omega_{2}, \mathscr{A}_{2}, P_{2}\right)$ be two probability spaces. For $R^{n}$ or $C^{n}$ valued measurable functions $F, G$ defined on $\Omega_{1}, \Omega_{2}$ respectively, set: 


$$
\begin{array}{llll}
\mu(B)=P_{1} F^{-1}(B) & B \in R^{n} & \text { or } & C^{n} \\
\nu(B)=P_{2} G^{-1}(B) & B \in R^{n} & \text { or } & C^{n}
\end{array}
$$

so that $\mu$ and $\nu$ are the distribution functions of $F$ and $G$ under $P_{1}$ and $P_{2}$ respectively. Changes in $n, R, C, F$ or $G$, result perhaps in different $\mu$ and $\nu$; but to simplify our notation we will continue using the same symbols $\mu$ and $\nu$ in all cates. If

$$
\int\left|1+\sum_{i=1}^{n} a_{i} f_{i}\right|^{p} d P_{1}=\int\left|1+\sum_{i=1}^{n} a_{i} g_{i}\right|^{p} d P_{2},
$$

$0<p<+\infty, p \neq$ even integer, for all complex numbers $a_{1}, a_{2}, \cdots, a_{n}$, then [5], $F=\left(f_{1}, f_{2}, \cdots, f_{n}\right)$ and $G=\left(g_{1}, g_{2}, \cdots, g_{n}\right)$ have the same distribution, that is $\mu=\nu$.

In Theorem 3.2 of this section, we give a probabilistic proof of this result. (Real versions will be given in Theorem 3.1. In $\S 4$ we comment on further results.) The proof here is, more or less, Markov-potential theoretic.

Theorem 3.1. Let $F=\left(f_{1}, f_{2}, \cdots, f_{n}\right), G=\left(g_{1}, g_{2}, \cdots, g_{n}\right)$ be two $R^{n}$ valued measurable functions defined on $\Omega_{1}, \Omega_{2}$ respectively. If

$$
\int\left|1+\sum_{i=1}^{n} a_{i} f_{i}\right|^{p} d P_{1}=\int\left|1+\sum_{i=1}^{n} a_{i} g_{i}\right|^{p} d P_{2},
$$

$0<p<+\infty, p \neq$ even integer, for all reals $a_{1}, a_{2}, \cdots, a_{n}$. Then $\mu=\nu$.

Proof. Let $n=1$. Passing from $P_{1}, P_{2}$ to $\mu, \nu$ respectively,

$$
\int|1+a y|^{p} d \mu(y)=\int|1+a y|^{p} d \nu(y),
$$

$a^{7}$ is real which implies $\int|x-y|^{p} d \mu(y)=\int|x-y|^{p} d \nu(y)$ for $x$ real. Depending on $p$ we have the following cases:

(a) $0<p<1$ : So we may take $p=\alpha-1,1<\alpha<2$. Thus for $f=V \phi$ in Section 2 and Example (2),

$$
\begin{aligned}
\int f(y) d \mu(y) & =C \iint|x-y|^{\alpha-1} \phi(x) d x d \mu(y) \\
& =C \iint|x-y|^{\alpha-1} \phi(x) d \mu(y) d x \\
& =C \iint|x-y|^{\alpha-1} \phi(x) d \nu(y) d x \\
& =\int f(y) d \nu(y)
\end{aligned}
$$


for every $f \in \mathscr{D}(A)$, proving $\mu=\nu$ by denseness of $\mathscr{D}(A)$.

(b) $p=1$ : Here we use Example (1), and follow the procedure of the case just preceded.

(c) $1<p<2$ : So that $p=\alpha+1,0<\alpha<1$. For $f \in C_{k}^{\infty}(S)$

$$
\int V f(y) d \mu(y)=\iint|x-y|^{\alpha-1} f(x) d x d \mu(y), \quad 0<\alpha<1,
$$

by Example (2). Integrating twice by parts relative to $x$, the right hand side is now equal to $\iint|x-y|^{\alpha+1} f^{\prime \prime}(x) d x d \mu(y)$. Repeating the same operations for $\nu, \int V f(y) d \mu(y)=\int V f(y) d \nu(y)$, implying $\mu=\nu$.

(d) $2<p<+\infty, p \neq$ even integer: Here we take an even number of derivatives or use integration by parts and reduce this case to one of the preceding cases.

Finally, if $n>1$ and

$$
\int\left|1+\sum_{1}^{n} a_{i} x_{i}\right|^{p} d \mu(x)=\int\left|1+\sum a_{i} x_{i}\right|^{p} d \nu(x),
$$

then it is obvious that $\int\left|1+a \sum_{i=1}^{n} a_{i} x_{i}\right|^{p} d \mu=\int\left|1+a \sum_{i=1}^{n} a_{i} x_{i}\right|^{p} d \nu$ which by $n=1$ case implies that $\sum_{i=1}^{n} a_{i} f_{i}$ and $\sum_{i=1}^{n} a_{i} g_{i}$ have the same distribution for all reals $a_{1}, a_{2}, \cdots, a_{n}$. Thus $F$ and $G$ have the distribution; that is $\mu=\nu$.

THEOREM 3.2. Let $F$ and $G$ be $C^{n}$ valued measurable functions on $\Omega_{1}, \Omega_{2}$ respectively. If

$$
\int\left|1+\sum_{i=1}^{n} c_{i} f_{i}\right|^{p} d P_{1}=\int\left|1+\sum_{i=1}^{n} c_{i} g_{i}\right|^{p} d P_{2},
$$

$0<p<+\infty$ and $p \neq$ even integer, for all complex numbers $c_{1}, c_{2}, \cdots, c_{n}$. Then $\mu=\nu$.

Proof. We identify $C$ with $R^{2}$, and start as before with $n=1$. Thus $\int|x-y|^{p} d \mu(x)=\int|x-y|^{p} d \nu(y)$ with $x=x_{1}+i x_{2}, y=y_{1}+i y_{2}$. Recalling [1] that the Markov process at hand has Green's potential, we have for $f \in C_{k}^{+\infty}(S)$

$$
\iint|x-y|^{p} f(x) d x d \mu(y)=\iint|x-y|^{p} f(x) d x d \nu(x),
$$

using the hypothesis. There is no problem of integrability, since the hypothesis implies continuity in $x$. Integrate by parts twice relative to $x_{1}$, repeat the same for $x_{2}$, and add up. Performing this operation an appropriate number of times 


$$
\iint|x-y|^{p-2 k} g(x) d x d \mu(y)=\iint|x-y|^{p-2 k} g(x) d x d \nu(y)
$$

and in such a way that $-2<p-2 k<0$ or

$$
\alpha-2=p-2 k \text { and } 0<\alpha<2 \text {. }
$$

Example (3) completes the proof for $n=1$. If $n>1$ the proof is similar to that of $n>1$ in Theorem 3.1 .

4. Remarks. (1) Theorems 3.1 and 3.2 may be extended to $-\infty<p<0$ in the sense of analytic continuation of $\alpha$, a procedure well known in Riesz potential theory.

(2) As an application of Theorem 3.1 one may prove a real version of Theorem 2 of [5].

(3) The case $p=1$ of Theorem 3.1 can be proved in an elementary fashion. Consider for an arbitrary positive $x$,

$$
\int|x+\alpha y| d \mu(y)=\int|x+\alpha y| d \nu(y)
$$

Differentiate relative to $\alpha$ at $\alpha=0$, obtain by Lebesgue's dominated convergence theorem $\int y d \mu(y)=\int y d \nu(y)$. Combine this with the hypothesis $\int(z-y)^{+} d \mu(y)=\int(z-y)^{+} d \nu(y)$. Integrate both sides then differentiate relative to $z$, and obtain $\mu=\nu$.

\section{REFERENCES}

1. R. M. Blumenthal and R. K. Getoor, Markov Processes and Potential Theory, Academic Press (1968).

2. E. B. Dynkin, Markov Process, Vol. 1, Academic Press (1965).

3. K. Ito and H. P. McKean, Jr., Diffusion Processes and Their Sample Paths, Academic Press (1965).

4. S. C. Port, Potentials Associated with Recurrent Stable Markov Processes and Potential Theory, John Wiley and Sons (1967).

5. W. Rudin, $L^{p}$-isometries and equimeasurability, Indiana University Math. J., (1976).

6. K-I. Sato, Potential operators for Markov processes, Sixth Berkeley Symposium on Math. Stat. and Probability, Vol. III, (1970).

7. - Core of potential operators for processes with stationary independent increments, Nagoya Math. J., 48 (1972), 129-145.

8. K. Yosida, The existence of potential operator associated with an equicontinuous semigroup of class $C_{0}$, Studia Math., 31 (1968).

9. - On the potential operators associated with Brownian motions, J. Analyse Math., 23 (1970).

Received May 20, 1977.

UNIVERSITY OF AlBerta

Edmonton, Alberta, Canada

T6G 2G1 



\section{PACIFIC JOURNAL OF MATHEMATICS}

\section{EDITORS}

RICHARD ARENS (Managing Editor)

University of California

Los Angeles, California 90024

C. W. CURTIS

University of Oregon

Eugene, OR 97403

C. C. MOORE

University of California

Berkeley, CA 94720

\section{J. DUGUNDJI}

Department of Mathematics University of Southern California Los Angeles, California 90007

R. Finn aNd J. Milgram Stanford University Stanford, California 94305

\section{ASSOCIATE EDITORS}

E. F. BeCKenbaCH

B. H. NeumanN

F. WOLF

K. YosHIDA

\section{SUPPORTING INSTITUTIONS}

UNIVERSITY OF BRITISH COLUMBIA CALIFORNIA INSTITUTE OF TECHNOLOGY UNIVERSITY OF CALIFORNIA MONTANA STATE UNIVERSITY UNIVERSITY OF NEVADA, RENO NEW MEXICO STATE UNIVERSITY OREGON STATE UNIVERSITY UNIVERSITY OF OREGON
UNIVERSITY OF SOUTHERN CALIFORNIA STANFORD UNIVERSITY UNIVERSITY OF HAWAII UNIVERSITY OF TOKYO UNIVERSITY OF UTAH WASHINGTON STATE UNIVERSITY UNIVERSITY OF WASHINGTON 


\section{Pacific Journal of Mathematics}

\section{Vol. 76, No. $1 \quad$ November, 1978}

Ata Nuri Al-Hussaini, Potential operators and equimeasurability ......... 1

Tim Anderson and Erwin Kleinfeld, Semisimple nil algebras of type $\delta . \ldots .99$

Stephen LaVern Campbell, Linear operators for which $T^{*} T$ and $T+T^{*}$

commute. III ......................................

Robert Jay Daverman, Special approximations to embeddings of codimension one spheres...............................

Donald M. Davis, Connective coverings of $\mathrm{BO}$ and immersions of projective

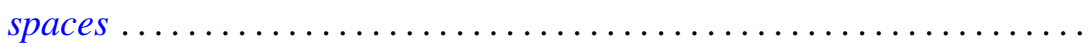

V. L. (Vagn Lundsgaard) Hansen, The homotopy type of the space of maps of

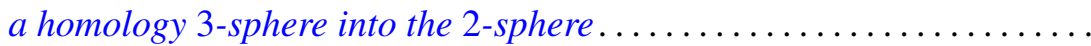

James Victor Herod, A product integral representation for the generalized

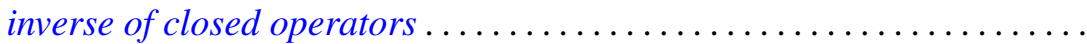

A. A. Iskander, Definability in the lattice of ring varieties ..............

Russell Allan Johnson, Existence of a strong lifting commuting with a

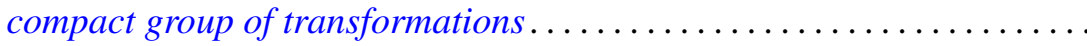

Heikki J. K. Junnila, Neighbornets...................... 83

Klaus Kalb, On the expansion in joint generalized eigenvectors . ......... 109

F. J. Martinelli, Construction of generalized normal numbers . . . . . . . . . 117

Edward O'Neill, On Massey products ....................... 123

Vern Ival Paulsen, Continuous canonical forms for matrices under unitary

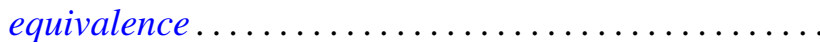

Justin Peters and Terje Sund, Automorphisms of locally compact groups . . . 143

Duane Randall, Tangent frame fields on spin manifolds . . . .

Jeffrey Brian Remmel, Realizing partial orderings by classes of co-simple sets . . . .

J. Hyam Rubinstein, One-sided Heegaard splittings of 3-manifolds ...

Donald Charles Rung, Meier type theorems for general boundary approach

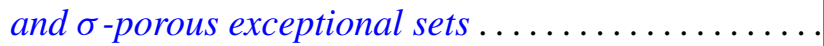

Ryōtarō Satō, Positive operators and the ergodic theorem

Ira H. Shavel, A class of algebraic surfaces of general type constructed from

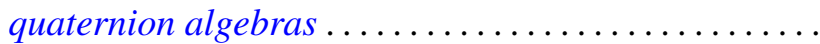

Patrick F. Smith, Decomposing modules into projectives and injectives ....

Sergio Eduardo Zarantonello, The sheaf of outer functions in the polydisc... 